\title{
Alhazen and the Nearest Neighbor Rule
}

\author{
Marcello Pelillo \\ DAIS, Università Ca' Foscari di Venezia \\ Via Torino 155, 30172 Venezia Mestre, Italy \\ E-mail: marcello.pelillo@unive.it
}

\begin{abstract}
We show that a clear formulation of the nearest neighbor decision rule for pattern classification can be found, along with other remarkably modern ideas, in an influential medieval treatise on visual perception authored by Alhazen, one of the major figures in the so-called "Islamic golden age." To put the work in context we provide also a brief description of some of the salient points of Alhazen's theory.

Keywords: Alhazen, Nearest neighbor classification, Visual perception, History of science.
\end{abstract}

\section{Introduction}

This is how Thomas Cover, many years later, detailed the origins of the nearest neighbor $(\mathrm{NN})$ decision rule for pattern classification:

Early in 1966 when I first began teaching at Stanford, a student, Peter Hart, walked into my office with an interesting problem. He said that Charles Cole and he were using a pattern classification scheme which, for lack of a better word, they described as the nearest neighbor procedure. This scheme assigned to an as yet unclassified observation the 
classification of the nearest neighbor. Were there any good theoretical properties of this procedure? (Cover, 1982)

Eventually, after several afternoon meetings, they were able to prove that the probability of error of this simple classification rule is bounded above by twice the Bayes minimum probability of error, and published one of the most influential papers in pattern recognition ${ }^{1}$ (Cover and Hart, 1967). Nearest neighbor search, sometimes referred to as the "post office problem" (Knuth, 1973), arises also as a fundamental problem in a variety of computer science areas ranging from information retrieval to computational geometry and coding theory - see (Papadopoulos and Manolopoulos, 2005) for a database perspective.

Although, as pointed out by Cover himself, the basic principle motivating the NN rule, namely that "things that look alike must be alike," is lost in the mists of time (traces of which can in fact be found already in the earliest extant philosophical fragments), we show in this paper that a neat, explicit formulation of the $\mathrm{NN}$ rule as a classification procedure can be found in a little known part of an otherwise enormously influential medieval treatise which paved the way for the later development of modern visual perception theories.

The author of this treatise was Abu Ali al-Hasan ibn al-Hasan ibn alHaytham, better known in the West as Alhazen, who ranks among the most prominent figures in medieval Islamic science. He flourished in Egypt in

\footnotetext{
${ }^{1}$ In 1998 the paper received a Golden Jubilee Paper Award from the IEEE Information Theory Society.
} 
the early eleventh century and wrote extensively on various topics including physics, astronomy, and mathematics. The most influential of his writings, however, is undoubtedly the Kitab al-Manazir (or "Book of Optics"), which was written probably around the 1030's and translated into Latin in the late twelfth or early thirteenth century under the title De Aspectibus or Perspectiva.

Influenced by Ptolemy's optical theory, Alhazen's achievements in the field of visual perception are astonishing and were until recently neglected by the modern Western tradition. He anticipated by centuries many fundamental ideas that are still alive today such as, for example, Helmholtz's principle of unconscious inference, the apparent distance account of the moon illusion, the role of eye movements in visual perception, and he is also regarded as a precursor of the scientific method. Howard (1996) counted as many as eleven such anticipations and provided a detailed account of their development.

In this paper, we add one more item to the list. In fact, as it turns out, an important, though neglected, component of Alhazen's theory was a simple classification mechanism which is essentially identical to Cover and Hart's NN rule. We take this opportunity to make some of Alhazen's remarkable ideas on visual recognition known within the pattern recognition community. In particular, in Section 2 we provide a brief summary of Alhazen's optical theory, while in Section 3 we describe the psychological component of his account (where, as we shall see, we encounter the NN classification rule). Finally, in Section 4, we offer some speculations concerning the originality of Alhazen's proposal. 


\section{Alhazen's Optical Model}

Of the seven books which compose Alhazen's treatise, the one which had the most durable impact on subsequent thinkers was mainly the first, which deals with such topics as the propagation of light, the anatomy of the eye, the visual pathways, etc., and expounds a systematic optical theory that was to put an end to long-lasting controversies. Indeed, for several centuries various rival doctrines of light and vision coexisted side by side, a state of affairs which motivated Kuhn (1970) to use this as an illustrative example of what he called a "pre-paradigmatic" stage of a scientific theory. Followers of the Euclidean and Ptolemaic traditions defended an extramission doctrine which postulates the existence of visual rays emanating from one's eye; the adherents of the atomistic school maintained an opposite view according to which thin replicas of the visible bodies emanate continually in all directions to enter the observer's eye. And there were of course variations of the basic themes such as Plato's combined extramission-intromission theory, and Aristotle's intromission version which insisted on the changes produced by the visible bodies on a transparent medium (Ronchi, 1952; Lindberg, 1976; Wade, 1998).

Alhazen would have none of this. Using a variety of ingenious arguments, including the phenomenon that today we call afterimage, he commences the first book of his De Aspectibus by providing compelling evidence against the extramission theory. He therefore sided with the intromission camp, but the theory he developed was radically different from the previous ones.

As pointed out by Lindberg (1976), Alhazen drew together the three optical traditions of his time, the mathematical, the anatomical and the physical, 
thereby creating a single comprehensive theory. The most revolutionary part of his theory, however, is neither the anatomical (which, as he himself admitted, was essentially that of Galen ${ }^{2}$ ), nor the mathematical. Instead, it was his physical explanations of optical phenomena, and in particular his punctiform analysis of visible bodies, that had the most lasting impact. Roughly, according to Alhazen's theory the surfaces of visible objects are thought of as composed of minute patches (points) which, when illuminated by a visual source, radiate their image rectilinearly in all directions. Some of these radiations enter the observer's eye through the pupil, wherever they meet, thereby giving rise to a series of processes which culminate in the experience of visual perception - see (Russell, 1996) for a more accurate description of the image formation process. Note that this intromissionist account of optical phenomena differs markedly from the preceding ones because, contrary to tradition, here it is not the body, taken as a whole, but each of its constituent points from which visual form issues. This might sound like today's textbook explanation, but we have to wait until Kepler to get the first truly modern optical theory.

The main problem Alhazen had to face was of course to explain how this collection of tiny images are reassembled into the eye to get a coherent picture of external objects. Note that, following Galen, he believed that the sensitive organ within the eye was not the retina but the crystalline, which was thought to be in a more central position than it actually is. Alhazen understood image formation in the eye in terms of a pinhole camera, of which

\footnotetext{
${ }^{2}$ In fact, he did not have a chance to do anatomical observations as Muslim's tradition forbade the dissection of the human body.
} 
he is credited to be the inventor, but realized that the pupil is too large to allow the eye to work precisely that way, as visual rays would intermingle and confusion would therefore arise. Since at the time of his writing he lacked a clear understanding of how lens work, a topographic, point-to-point correspondence between the visual field and the crystalline was difficult to establish. Alhazen's ingenious solution to this problem is recognized to be one of his greatest achievements in vision. He understood the importance of the role played by refraction in image formation and contended that, of all the visual rays emanating from a single point of a body's surface, only the one which hits the cornea perpendicularly, and hence is not bent by refraction, contributes to the formation of the image in the interior of the eye. In fact, Alhazen believed that refracted rays must lose their power to stimulate the sensitive organ, a principle that is reminiscent of what is known today as the Stiles-Crawford effect.

Alhazen's ideas were largely ignored for about 250 years, but they even-

tually came to dominate Western optical thought up to the beginning of the seventeenth century, deeply influencing scientists and philosophers such as Roger Bacon, John Pecham, and Witelo, only to fall again into oblivion until its recent rediscovery (Lindberg, 1976; Wade, 1998).

\section{Beyond Optics: Visual Recognition as Nearest Neighbor Search}

Some six centuries after Alhazen, and indirectly influenced by him, Kepler provided the first correct explanations of the mechanisms underlying image formation in the eye, and claimed he had essentially solved the problem of vision (or, at least, that it was not his business to investigate further). As 
he put it: "I say that vision occurs when the image of the whole hemisphere of the world that is before the eye [...] is fixed on the reddish white concave surface of the retina" (Lindberg, 1976, p. 203).

Following a tradition which goes back to Aristotle and to some extent to Galen, Alhazen held a more sophisticated view. In fact, after explaining the basic mechanisms of image formation, towards the end of Book I he hastens to say:

in terms of naked sensation, sight perceives only the light and color that are in the visible object. The remaining characteristics of visible objects that sight perceive, e.g., shape, size, and the like, are perceived by sight not through naked sensation but through reason and defining features. And we shall show this later in the second book [... ${ }^{3}$ (Smith, 2001, p. 374)

It is indeed the second book of his treatise, which has typically been neglected by ancient and modern scholars alike (Sabra, 1978; Howard, 1996), which concerns us the most as it is here that we find a detailed exposition of the NN rule for classification, together with a series of remarkably modern observations about the nature of visual cognition. The account provided in this book can be considered in all regards as the first modern psychological theory of visual perception.

In Alhazen's theory, vision unfolds basically in three stages of increasing

\footnotetext{
${ }^{3}$ All quotations from Alhazen's treatise are taken from a recent English translation of the Latin edition De Aspectibus, which is more readily accessible to the non-specialist than the classical Arabic-to-English counterpart (Sabra, 1989).
} 
abstraction and complexity, starting from the passive registration of light and color in the eye. As he put it:

of [all] the characteristics that are perceived by visual sensation, some are perceived through brute sensation, some through recognition, and some through judgment and differentiation. (Smith, 2001, p. 433)

Of these stages, it is the second which concerns us here as it is the faculty of recognition, and nothing else, which is responsible for visual categorization:

Sight also perceives many things by means of recognition, so it recognizes that a human is a human, that a horse is a horse, and that Socrates is Socrates when it has seen the same thing before. (Smith, 2001, p. 431)

and then:

Sight perceives what kind of thing a visible object is through recognition exclusively. (Smith, 2001, p. 431)

But, what is exactly this faculty of recognition, and how does it work? Alhazen's response leaves no room for doubt. In the first place, he makes it clear that recognition is different from mere sensation as it involves memory and "imagination" (which is the storehouse of remembered perceptions from which memory itself recovers them). In fact:

recognition is the perception of similarity between two forms - i.e., of the form sight perceives at the moment of recognition and the form of that visible object, or its like, that it has perceived one or more times before. (Smith, 2001, p. 432) 
Therefore, since it involves perception of similarity, recognition is a form of judgment, but:

this form of judgment is distinct from other [forms of] judging, because, rather than involving an evaluation of all the characteristics of the form, recognition will occur through defining features. (Smith, 2001, p. 432)

This is an important point as it marks a distinction between unconscious and conscious inferences. The "other forms of judging" Alhazen had in mind, in fact, are those involving visual scrutiny (i.e., eye movement) and thus take some time. The inferential processes underlying recognition, instead, get unnoticed as they are performed in an extraordinarily short time by a few "defining features," which correspond basically to the essential (as opposed to the accidental) properties that are shared by members of a given category.

Alhazen offered a surprisingly modern, purely empiricist, account of the way in which categories (or, in his terminology, "universal forms") do arise in one's "soul." As he put it:

That universal forms of visible aspects occur in the soul and are impressed in the imagination is due to the fact that there are certain kinds of visible characteristics, such as form or shape, according to which individuals of a certain kind will be identical, whereas those individuals vary according to [other] particular characteristics that are perceived by the sense of sight. (Smith, 2001, p. 518)

and then:

It is thus through the effect of perceiving individuals of the same kind by sight that the universal form of their kind will recur [in the soul] 
along with the various particular forms of those individuals. (Smith, 2001, p. 518)

Hence, in Alhazen's account, universal forms are "impressed in the imagination" by the repeated perceptions of individuals belonging to the same category, and consist simply of a collection of the particular properties they all have in common.

Having clarified the nature of recognition and the role played by memory and similarity, Alhazen had all the ingredients to elucidate the mechanisms of visual categorization:

Hence, when sight perceives some visible object, the faculty of discrimination immediately seeks its counterpart among the forms persisting in the imagination, and when it finds some form in the imagination that is like the form of that visible object, it will recognize that visible object and will perceive what kind of object it is. (Smith, 2001, p. 519)

which is a surprisingly clear, almost algorithmic, exposition of Cover and Hart's NN classification rule. Interestingly, he also considered the case when the perceived object has no similar counterpart in memory:

But if it does not find a form similar to the form of that visible object among the forms persisting in the imagination, it will not recognize that visible object or perceive what kind of thing it is. (Smith, 2001, p. 519)

this being but an informal way of endowing the classification rule with a "reject option" (Hellman, 1970). 
As pointed out by Sabra (1978), a remarkable feature of Alhazen's account of visual recognition is that nowhere in his explanation does he need to make recourse to the "agent intellect" or other ambiguous entities of Aristotelian flavor, thereby markedly distinguishing him from his predecessors.

\section{Conclusions}

We have brought to the attention of the pattern recognition community that a clear exposition of the well-known nearest neighbor rule for classification can be found in an little-known part of Alhazen's influential treatise on optics, written in Egypt in the beginning of the eleventh century. The rule is a component of a psychological account of visual recognition involving high-level faculties such as judgment and syllogistic inference which has been almost totally neglected by subsequent thinkers.

An obvious question that arises is whether Alhazen took this idea from earlier scholars or, in any case, whether other manifestations of the NN rule were put forward in the period before Alhazen. There are reasons to believe that this is not the case. The study of optics in antiquity was mainly a matter of mathematics, physiology and physics, and little or no attention was given to the psychological processes underlying vision. ${ }^{4}$ As one of the most prominent scholars of Alhazen has recently remarked: "in and of itself the new level of emphasis on psychology in Ibn al-Haytham's Optics was historically an important development that has recently attracted the attention of psy-

\footnotetext{
${ }^{4}$ Indeed, as pointed out by Prof. A. Mark Smith in a personal communication, Ptolemy also brought to some extent psychology into the visual process, but he did not provide the elaborate, empirical account that Alhazen did.
} 
chologists and historians of psychology. The emphasis allowed him to open a new chapter in the history of the systematic study of vision" (Sabra, 2003, p. 110). Some ancient thinkers, most notably Galen and his followers, did indeed realize that vision cannot end with the reception of forms in the eye and does require further processing by the brain, but they did not venture into an explicit description of the underlying mechanisms (Lindberg, 1976). In fact, as we noted above, as late as in the seventeenth century Kepler was reluctant to speculate on the nature of the post-retinal operations of visual perception (although his contemporary Descartes was not), finding it even difficult to explain how we see an upright world from an inverted retinal image.

In order to be able to suggest an NN-like classification rule with such clarity as Alhazen's own formulation, one has to have a clear idea of the role played by memory, similarity and, above all, inference in vision. Although some of these ingredients were already present in Greek philosophy, the notion of perception as an inferential process was to a large extent lacking in antiquity before Alhazen, and was in fact one of his most remarkable anticipations. It is to be conjectured, therefore, that Alhazen's is the first explicit formulation of the NN classification algorithm on record.

\section{Acknowledgments}

I am indebted to the following people who read a preliminary version of the paper, provided useful comments and encouraged me to submit it: Joachim Buhmann, Horst Bunke, Terry Caelli, Tiberio Caetano, Bob Duin, Ian Howard, Jan Koenderink, Walter Kropatsch, Zyg Pizlo, Fabio Roli, 
Teresa Scantamburlo, Nick Wade, and Steve Zucker. In particular, I am grateful to Gül Russell and Mark Smith, whose meticulous historical remarks allowed me to fix a few inaccuracies.

\section{References}

Cover, T.M., 1982. This week's citation classic. Current Contents 13, 20.

Cover, T.M., Hart, P.E., 1967. Nearest neighbor pattern classification. IEEE Transactions on Information Theory 13, 21-27.

Hellman, M.E., 1970. The nearest neighbor classification rule with a reject option. IEEE Transactions on Systems Science and Cybernetics 6, 179185.

Howard, I.P., 1996. Alhazen's neglected discoveries of visual phenomena. Perception 25, 1203-1217.

Knuth, D.E., 1973. The Art of Computer Programming - Vol III: Sorting and Searching. Addison Wesley, Reading, MA.

Kuhn, T.S., 1970. The Structure of Scientific Revolutions. The University of Chicago Press, Chicago. 2nd edition.

Lindberg, D.C., 1976. Theories of Vision from Al-Kindi to Kepler. The University of Chicago Press, Chicago.

Papadopoulos, A.N., Manolopoulos, Y., 2005. Nearest Neighbor Search: A Database Perspective. Springer, New York. 
Ronchi, V., 1952. Storia della luce. Zanichelli, Bologna. English translation: The Nature of Light: An Historical Survey, Harvard University Press, Cambridge, MA, 1970.

Russell, G.A., 1996. The emergence of physiological optics, in: Rashed, R., (Ed.), Encyclopedia of the History of Arabic Science, Vol. 2. Routledge, London, UK, pp. 672-715.

Sabra, A.I., 1978. Sensation and inference in Alhazen's theory of visual perception, in: Machamer, P.K., Turnbull, R.G. (Eds.), Studies in Perception. Ohio State University Press, Columbus, pp. 160-185.

Sabra, A.I., 1989. The Optics of Ibn al-Haytham. The Warburg Institute, London, UK.

Sabra, A.I., 2003. Ibn al-Haytham's revolutionary project in optics: The achievement and the obstacle, in: Hogendijk, J.P., Sabra, A.I. (Eds.), The Enterprise of Science in Islam: New Perspectives. MIT Press, Cambridge, MA, pp. 85-118.

Smith, A.M., 2001. Alhacen's Theory of Visual Perception. American Philosophical Society, Philadelphia.

Wade, N.J., 1998. A Natural History of Vision. MIT Press, Cambridge, MA. 